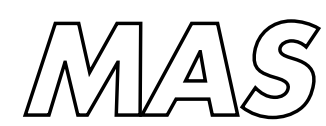

Modelling, Analysis and Simulation

Modelling, Analysis and Simulation
MAS Convergent asymptotic expansions of Charlier, Laguerre
and Jacobi polynomials

José L. López, Nico M. Temme

Report MAS-E0320 December 16, 2003 
CWI is the National Research Institute for Mathematics and Computer Science. It is sponsored by the Netherlands Organization for Scientific Research (NWO).

CWI is a founding member of ERCIM, the European Research Consortium for Informatics and Mathematics.

CWI's research has a theme-oriented structure and is grouped into four clusters. Listed below are the names of the clusters and in parentheses their acronyms.

Probability, Networks and Algorithms (PNA)

Software Engineering (SEN)

Modelling, Analysis and Simulation (MAS)

Information Systems (INS)

Copyright (C) 2003, Stichting Centrum voor Wiskunde en Informatica

P.O. Box 94079, 1090 GB Amsterdam (NL)

Kruislaan 413, 1098 SJ Amsterdam (NL)

Telephone +31205929333

Telefax +31205924199

ISSN 1386-3703 


\title{
Convergent asymptotic expansions of Charlier, Laguerre and Jacobi polynomials
}

\begin{abstract}
Convergent expansions are derived for three types of orthogonal polynomials: Charlier, Laguerre and Jacobi. The expansions have asymptotic properties for large values of the degree. The expansions are given in terms of functions that are special cases of the given polynomials. The method is based on expanding integrals in one or two points of the complex plane, these points being saddle points of the phase functions of the integrands.
\end{abstract}

2000 Mathematics Subject Classification: 33C45, 41A60, 30E20

Keywords and Phrases: Charlier polynomials, Laguerre polynomials, Jacobi polynomials, asymptotic expansions, saddle point methods, two-points Taylor expansions.

Note: This paper has been accepted for publication in the Proceedings A (Mathematics) of the Royal Society of Edinburgh. 


\title{
Convergent asymptotic expansions of Charlier, Laguerre and Jacobi polynomials
}

\author{
José L. López ${ }^{1}$ and Nico M. Temme ${ }^{2}$ \\ ${ }^{1}$ Departamento de Matématica e Informática, Universidad Pública de Navarra, \\ 31006-Pamplona, Spain (j1.lopez@unavarra.es), \\ ${ }^{2}$ CWI, P.O. Box 94079, 1090 GB Amsterdam, The Netherlands (nicot@cwi.nl).
}

\begin{abstract}
Convergent expansions are derived for three types of orthogonal polynomials: Charlier, Laguerre and Jacobi. The expansions have asymptotic properties for large values of the degree. The expansions are given in terms of functions that are special cases of the given polynomials. The method is based on expanding integrals in one or two points of the complex plane, these points being saddle points of the phase functions of the integrands.
\end{abstract}

2000 Mathematics Subject Classification: 33C45, 41A60, 30E20.

Keywords \& Phrases: Charlier polynomials, Laguerre polynomials, Jacobi polynomials, asymptotic expansions, saddle point methods, two-points Taylor expansions.

\section{Introduction}

In a previous paper [9] we have studied the expansion of an analytic function at two finite points in the complex plane. The domain of convergence is a Cassini oval around the two points. The main motivation for that paper was to obtain the coefficients of asymptotic expansions of certain integrals. In the present paper we give a few examples in which the expansion of an integral at two saddle points yields a convergent expansion that has an asymptotic property for large values of a parameter.

In the well-known methods for deriving asymptotic expansions of integrals a basic step is transforming the integral into a standard form, and the transformation usually gives a new integral in which the integrand contains implicitly defined functions that are difficult to handle. In the method of this paper we avoid a transformation and, in addition, we derive convergent expansions.

We start with a simple example in which only one saddle point occurs, and in which a function is expanded at that saddle point. This gives an expansion for the Charlier polynomials. 
In two other examples (Laguerre and Jacobi polynomials) we take into account two saddle points, and again two convergent expansions can be constructed with the desired property. The approximants belong to the same class of polynomials as the original ones, but they are of a simpler type (Hermite and Chebyshev, respectively). The asymptotic property follows from recursion relations for functions appearing in the expansions. The convergence follows from the fact that an integral along a finite contour is expanded inside a domain of uniform convergence.

In the examples given in this paper the contour integrals are based on Cauchy-type integrals obtained from generating functions. When the contour is finite a proof of the convergence is usually rather easy. For more general finite contours and more general integrals we expect that the method can be applied as well. For example, we can apply the method to the Gauss hypergeometric function and the incomplete gamma function, with different integral representations.

Also, the methods of this paper can be generalized by considering Taylor expansions at more than two points. In [10] we give details on the theory of multi-point Taylor expansions, and in a future paper we will give details on applications to integrals with, for example, three saddle points.

We show a few graphs that indicate the nature of the approximations, and in a final section we mention a few examples in which other functions are considered.

\section{A simplified version of the saddle point method}

Throughout this paper we are concerned with finding asymptotic expansions of integrals of the form

$$
F(n) \equiv \int_{\Gamma} f(w) e^{n g(w)} \frac{d w}{w^{n+1}}
$$

where $f(w)$ and $g(w)$ are analytic in a domain $\Omega$ of the complex plane that contains the origin; $\Gamma$ is a circle with center at the origin and contained in $\Omega ; n$ is a large positive integer. We assume, as it usually happens to be the case, that the asymptotic behavior of the integral $F(n)$ for large $n$ is determined by contributions from the saddle points of $\varphi(w)=g(w)-\ln w[[15]$, Chap. 2, §4].

The standard saddle point method consists of

(i) deforming the contour of integration $\Gamma$ into a new path that crosses one or some of the saddle points of $\varphi(w)$;

(ii) a suitable change of the variable of integration;

(iii) application of Watson's lemma or Laplace's method.

Instead of applying the standard saddle point method, we will proceed in a simpler way: just substitute a power series expansion at one or more saddle points of the function $f(w)$ in $(2.1)$. If there is just one saddle point $w_{0}$, then that power series is its Taylor 
expansion at $w_{0}$ :

$$
f(w)=\sum_{k=0}^{\infty} \frac{f^{(k)}\left(w_{0}\right)}{k !}\left(w-w_{0}\right)^{k},
$$

which is uniformly convergent for $w$ in a disk $D_{r}\left(w_{0}\right) \equiv\left\{w \in \Omega,\left|w-w_{0}\right|<r\right\}$ with center at $w_{0}$ and radius $r=\operatorname{Inf}_{w \in \ell} \Omega\left(w-w_{0} \mid\right.$. If there are two saddle points $w_{1}$ and $w_{2}$, then that power series is its two-point Taylor series at $w_{1}$ and $w_{2}$ [9]:

$$
f(w)=\sum_{n=0}^{\infty}\left[a_{n}\left(w-w_{1}\right)+a_{n}^{\prime}\left(w-w_{2}\right)\right]\left(w-w_{1}\right)^{n}\left(w-w_{2}\right)^{n},
$$

where

$$
a_{0} \equiv \frac{f\left(w_{2}\right)}{w_{2}-w_{1}}, \quad a_{0}^{\prime} \equiv \frac{f\left(w_{1}\right)}{w_{1}-w_{2}}
$$

and, for $n=1,2,3, \ldots$,

$$
\begin{aligned}
& a_{n} \equiv \frac{1}{n !} \sum_{k=0}^{n} \frac{(n+k-1) !}{k !(n-k) !} \frac{(-1)^{n+1} n f^{(n-k)}\left(w_{2}\right)+(-1)^{k} k f^{(n-k)}\left(w_{1}\right)}{\left(w_{1}-w_{2}\right)^{n+k+1}}, \\
& a_{n}^{\prime} \equiv \frac{1}{n !} \sum_{k=0}^{n} \frac{(n+k-1) !}{k !(n-k) !} \frac{(-1)^{n+1} n f^{(n-k)}\left(w_{1}\right)+(-1)^{k} k f^{(n-k)}\left(w_{2}\right)}{\left(w_{2}-w_{1}\right)^{n+k+1}} .
\end{aligned}
$$

The expansion (2.3) is uniformly convergent for $w$ in a Cassini oval

$$
O_{r}\left(w_{1}, w_{2}\right) \equiv\left\{w \in \Omega,\left|w-w_{1}\right|\left|w-w_{2}\right|<r\right\}
$$

with foci at $w_{1}$ and $w_{2}$ and "radius" $r=\operatorname{Inf}_{w \in \ell \backslash \Omega}\left\{\left|w-w_{1}\right|\left|w-w_{2}\right|\right\}$; see [9].

If we substitute now (2.2) or (2.3) in (2.1) and interchange summation and integration we obtain an expansion of $F(n)$. This is proved in the following two propositions. Proposition 2.1. Let the right-hand side of (2.2) converge uniformly to $f(w)$ for $w \in D_{r}\left(w_{0}\right)$ with $\left|w_{0}\right|<r$, then

$$
F(n)=\sum_{k=0}^{\infty} \frac{f^{(k)}\left(w_{0}\right)}{k !} \int_{\Gamma}\left(w-w_{0}\right)^{k} e^{n \varphi(w)} \frac{d w}{w} .
$$

Proof. If $\left|w_{0}\right|<r$, then $0 \in D_{r}\left(w_{0}\right)$. Then we can choose a small enough circle $\Gamma$ in (2.1) such that $\Gamma \in D_{r}\left(w_{0}\right)$. Therefore, expansion (2.2) is uniformly convergent for $w \in \Gamma$. Introducing (2.2) in (2.1) and interchanging summation and integration we obtain (2.7).

Proposition 2.2. Let the right-hand side of (2.3) converge uniformly to $f(w)$ for $w \in O_{r}\left(w_{1}, w_{2}\right)$ with $\left|w_{1} w_{2}\right|<r$, then

$$
\begin{aligned}
F(n)= & \sum_{k=0}^{\infty} a_{k} \int_{\Gamma}\left(w-w_{1}\right)^{k+1}\left(w-w_{2}\right)^{k} e^{n \varphi(w)} \frac{d w}{w}+ \\
& \sum_{k=0}^{\infty} a_{k}^{\prime} \int_{\Gamma}\left(w-w_{1}\right)^{k}\left(w-w_{2}\right)^{k+1} e^{n \varphi(w)} \frac{d w}{w} .
\end{aligned}
$$


Proof. The proof is similar to that of Proposition 2.1.

In the remaining part of the paper we apply Proposition 2.1 or Proposition 2.2 to three specific examples of integrals $F(n)$ representing Charlier polynomials $C_{n}^{a}(n x)$, Laguerre polynomials $L_{n}^{\alpha}(n x)$ and Jacobi polynomials $P_{n}^{(\alpha, \beta)}(x)$. In this way, we obtain expansions of these polynomials for large values of $n$. In each example separately we prove that the corresponding expansions (2.7) or (2.8) are convergent in a certain region of the variable $x$ and that in fact, they have an asymptotic nature for large $n$, uniformly with respect to $x$ in certain domains of the region of convergence.

\section{Asymptotic expansions of Charlier polynomials in terms of Gamma func- tions}

The Charlier polynomials are defined by the generating function

$$
e^{-a w}(1+w)^{x}=\sum_{n=0}^{\infty} C_{n}^{a}(x) \frac{w^{n}}{n !},
$$

and have the explicit expression

$$
C_{n}^{a}(x)=\sum_{k=0}^{n}\left(\begin{array}{l}
n \\
k
\end{array}\right)\left(\begin{array}{l}
x \\
k
\end{array}\right) k !(-a)^{n-k} .
$$

The Charlier polynomials are orthogonal with respect to a discrete distribution on the positive real line. For an overview of properties, see [7]. Recent papers on asymptotics are [1], [2], [5], and [6]. In this section we give a simple convergent expansion of $C_{n}^{a}(x n)$, that has an asymptotic property for large $n$, uniformly for complex $a$ in compact sets and for complex $x$ bounded away from 1. A uniform expansion that holds for $x$ in a compact neighbourhood of $x=1$ is given in [1], where $J$-Bessel functions are used in the approximations. Also, uniform expansions that hold for $-\infty<x<\infty$ are given in $[2]$.

Theorem 3.1. For $x \neq 1, a \in \mathbb{C}$ and $n \in \mathbb{N}$, the Charlier polynomials have the expansion

$$
C_{n}^{a}(x n)=e^{a /(1-x)} \sum_{k=0}^{\infty} \frac{(-a)^{k}}{k !} \Phi_{k}(x, n)
$$

where

$$
\Phi_{0}(x, n) \equiv \frac{\Gamma(n x+1)}{\Gamma(n x+1-n)}, \quad \Phi_{1}(x, n) \equiv \frac{\Phi_{0}(x, n)}{(1-x)(n(x-1)+1)}
$$

and, for $k=0,1,2, \ldots$,

$$
\Phi_{k}(x, n) \equiv \frac{\Gamma(n x+1)}{\Gamma(n x-n+1)} \frac{1}{(1-x)^{k}}{ }_{2}^{2} F_{1}(-k,-n, n x-n+1 ; 1-x) .
$$


The sequence $\left\{\Phi_{k}(x, n), k=0,1,2, \ldots\right\}$ satisfies the recurrence

$$
\Phi_{k}(x, n)=\frac{1}{n(x-1)+k}\left[\frac{x(1-k)-k}{x-1} \Phi_{k-1}(x, n)+\frac{x(1-k)}{(x-1)^{2}} \Phi_{k-2}(x, n)\right],
$$

where $k=2,3, \ldots$ and is an asymptotic sequence for large $n$. For fixed $k$ we have

$$
\Phi_{k}(x, n)=\mathcal{O}\left(n^{-\lfloor(k+1) / 2\rfloor}(n|x|)_{n}\right)
$$

when $n \rightarrow \infty$, where $\lfloor\alpha\rfloor$ is the integer part of the real number $\alpha$. The asymptotic property holds uniformly with respect to complex $x,|x-1| \geq \delta>0$.

Observe that in the notation of Pochhammer's symbol $(a)_{n}$, defined by

$$
(a)_{0}=1, \quad(a)_{n}=\frac{\Gamma(a+n)}{\Gamma(a)}=a(a+1) \cdots(a+n-1), \quad n=0,1,2, \ldots,
$$

we have

$$
\Phi_{0}(x)=(-1)^{n} \frac{\Gamma(n-n x)}{\Gamma(-n x)}=(-1)^{n}(-n x)_{n}=(n x)(n x-1) \cdots(n x-(n-1)) .
$$

Proof. From (3.1) we derive the integral representation

$$
C_{n}^{a}(x)=\frac{n !}{2 \pi i} \int_{\Gamma} e^{-a w}(1+w)^{x} \frac{d w}{w^{n+1}}
$$

where $\Gamma$ is a circle with center at the origin and radius $<1$. We write this in the form

$$
C_{n}^{a}(n x)=\frac{n !}{2 \pi i} \int_{\Gamma} e^{-a w} e^{n \varphi(x, w)} \frac{d w}{w}
$$

where $\varphi(x, w) \equiv x \log (1+w)-\log w$. The only saddle point of $\varphi(x, w)$ is $w_{0}=(x-1)^{-1}$. The function $e^{-a w}$ is an entire function of $w$. Hence, the expansion

$$
e^{-a w}=\sum_{k=0}^{\infty} \frac{(-a)^{k} e^{-a w_{0}}}{k !}\left(w-w_{0}\right)^{k}
$$

is locally uniformly convergent for $w \in \mathbb{C}$ and $x \neq 1$. Therefore, after substituting this expansion in (3.10) and using Proposition 2.1 we obtain (3.3) with

$$
\Phi_{k}(x, n)=\frac{n !}{2 \pi i} \int_{\Gamma}\left(w-w_{0}\right)^{k}(1+w)^{x n} \frac{d w}{w^{n+1}} .
$$

To obtain the recurrence (3.6) we write

$$
\Phi_{k}(x, n)=\frac{(n-1) !}{2 \pi i} \frac{1}{x-1} \int_{\Gamma}\left(w-w_{0}\right)^{k-1}(1+w) \frac{\partial e^{n \varphi(x, w)}}{\partial w} d w
$$




$\begin{array}{ll}0.000000090 & 0.534449998 \\ 0.100006223 & 0.680932968 \\ 0.200157621 & 0.855641877 \\ 0.301812498 & 1.068772397 \\ 0.410358953 & 1.347867376\end{array}$

TABLE 3.2. The zeros of $C_{n}^{a}(n x)$ for $n=10$ and $a=1$.

Integrating by parts and performing a few straightforward manipulations we obtain (3.6). Equalities (3.4)-(3.5) follow after simple calculations. The asymptotic behavior in (3.7) for large $n$ follows from (3.4) and (3.6). From (3.4) we see that $\Phi_{0}=\mathcal{O}\left((n|x|)_{n}\right)$ (see formula (19) below) and that $\Phi_{1}=\mathcal{O}\left((n|x|)_{n} / n\right)$. Therefore, (3.7) is true for $k=0,1$. From here, the proof follows by induction over $k$. If (3.7) holds up to $k$, then $\Phi_{k-1}=\mathcal{O}\left(n^{-[k / 2]}(n|x|)_{n}\right)$ and $\Phi_{k}=\mathcal{O}\left(n^{-[(k+1) / 2]}(n|x|)_{n}\right)$. Using this in (3.6) with $k$ replaced by $k+1$ we have that $\Phi_{k+1}=\mathcal{O}\left(n^{-[(k+2) / 2]}(n|x|)_{n}\right)$.

Property (3.7) holds uniformly for $|x-1| \geq \delta>0$, also for complex $x$. Also, detailed information on the asymptotic behavior can easily be obtained from (3.12).

From (3.9) it follows that $\Phi_{0}(x)$ has $n$ zeros at $x=m / n, m=0,1, \ldots n-1\left(\Phi_{1}(x)\right.$ has the same zeros, except for $x=(n-1) / n)$. See also Table 3.2, where we give numerical values of the zeros of $C_{n}^{a}(n x)$ for $n=10$ and $a=1$. From the graphs in Figure 3.3 we also see that the early zeros are approximated quite well.

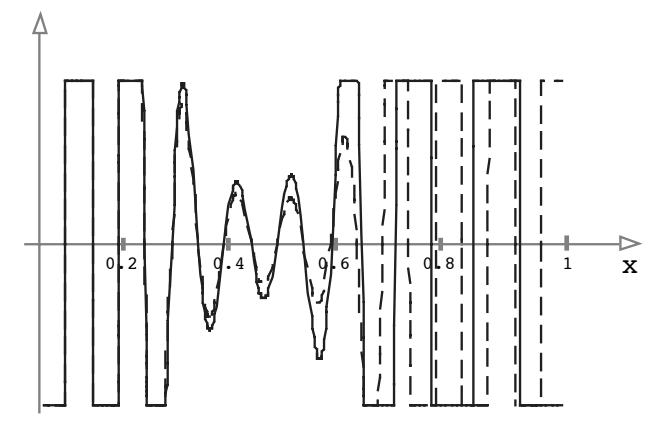

(a)

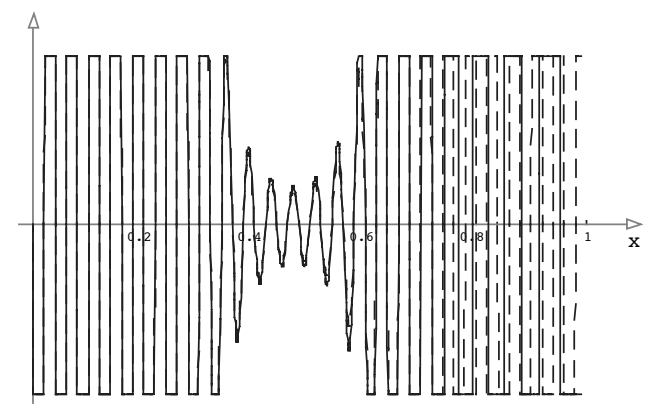

(b)

FiguRE 3.3. Numerical experiment on the approximation given in Theorem 3.1 for large $n$ and $x \in[0,1)$. Continuous lines represent the Charlier polynomial $C_{n}^{1}(n x)$ for (a) $n=20$ and (b) $n=50$. Dashed lines represent the first order approximation given by $e^{a /(1-x)} \Phi_{0}(x, n)$. Both graphics are cut for extreme values of the polynomials.

REMARK 3.4. When in expansion (3.11) the expansion point $w_{0}$ is not equal to the saddle point $(x-1)^{-1}$, we are not able to prove the asymptotic nature of expansion (3.3). This follows from the integration by parts procedure mentioned in the proof of 
Theorem 3.1. On the other hand, we can show (3.7) directly from the definition (3.12) with a change of variable like in the standard saddle point method.

\subsection{Details on the convergence}

It is of interest to verify the speed of convergence of the expansion in (3.3). We consider (3.12), with $k=\kappa n$, and determine the saddle point of $\left(w-w_{0}\right)^{\kappa}(1+w)^{x} w^{-1}$, where $\kappa$ is large. We consider $x$ fixed, and for $x<1$ we verify that a positive saddle point $w_{+}$occurs with $w_{+} \sim 1 /[(1-x) \kappa]$. There is a negative saddle point, whch is not relevant. We have

$$
\left[\left(w_{+}-w_{0}\right)^{\kappa}\left(1+w_{+}\right)^{x} w_{+}^{-1}\right]^{n} \sim \frac{\kappa^{n} e^{n}(1-x)^{n}}{(1-x)^{k}} .
$$

Multiplying this with $a^{k} n ! / k$ !, see (3.3) and (3.12), and using in Stirling's approximation of the factorials only the dominant parts, that is $k ! \sim(k / e)^{k}$, we see that the main information on $a^{k} n ! \Phi_{k}(k, n) / k$ ! is given by

$$
\frac{e^{k} k^{n} a^{k}(1-x)^{n}}{(1-x)^{k} k^{k}}
$$

where $k$ is large compared with $n$ and $x, x<1$. We see that the ratio of successive terms is about $a /[(1-x) k]$.

For other values of $x$, also complex, a similar analysis can be given, with some care in choosing the saddle points and defining the branches in the complex plane.

\begin{tabular}{|l|r|r|r|r|r|r|r|}
\hline$n$ & $C_{n}^{1}(0.25 n)$ & $\mathbf{C}_{n}^{a}(x, 0)$ & $\mathbf{C}_{n}^{a}(x, 1)$ & \multicolumn{1}{|c|}{$\mathbf{C}_{n}^{a}(x, 2)$} & $\mathbf{C}_{n}^{a}(x, 3)$ & $\mathbf{C}_{n}^{a}(x, 4)$ & $\mathbf{C}_{n}^{a}(x, 5)$ \\
\hline 10 & -1.03630 & -0.97736 & -1.02335 & -1.04747 & -1.00438 & -1.03633 & -1.0363 \\
30 & 4.35872 & 4.03823 & 4.28867 & 4.35077 & 4.35762 & 4.35858 & 4.35870 \\
50 & -4.86727 & -4.65813 & -4.82829 & -4.86464 & -4.86701 & -4.86725 & -4.86726 \\
90 & -2.94851 & -2.87926 & -2.93699 & -2.94808 & -2.94848 & -2.94851 & -2.94851 \\
\hline
\end{tabular}

TABLE 3.5. Numerical experiment on the convergence rate of expansion (3.3) for $x=.25$ and $a=1$. Here, $\mathbf{C}_{n}^{a}(x, N) \equiv e^{a /(1-x)} \sum_{k=0}^{N} \frac{(-a)^{k}}{k !} \Phi_{k}(x, n)$, represents the truncated series in (3.3). All the rows are multiplied by an appropriate constant in order to keep the numbers small.

\section{Asymptotic expansions of Laguerre polynomials in terms of Hermite poly- nomials}

The Laguerre polynomials can be defined by the generating function

$$
(1-t)^{-\alpha-1} e^{-t x /(1-t)}=\sum_{n=0}^{\infty} L_{n}^{\alpha}(x) t^{n}, \quad \alpha, x \in \mathbb{C}, \quad|t|<1 .
$$


and have the representation

$$
L_{n}^{\alpha}(x)=\sum_{k=0}^{n}(-1)^{k}\left(\begin{array}{c}
n+\alpha \\
n-k
\end{array}\right) \frac{x^{k}}{k !} .
$$

For deriving the asymptotic expansion we use the Cauchy integral that follows from (4.1):

$$
L_{n}^{(\alpha)}(x)=\frac{1}{2 \pi i} \int_{\Gamma} e^{x w /(w-1)}(1-w)^{-\alpha-1} \frac{d w}{w^{n+1}},
$$

where $\Gamma$ is a circle around the origin with radius $<1$. The many-valued functions $(1-w)^{\mu}$ appearing here and in the theorem assume the principal branch that is equal to 1 at $w=0$

The asymptotics for large $n$, fixed $\alpha$, is considered in [4]. For real $x$ two uniform expansions are given, one involving the $J$-Bessel function for $x$ in an interval that contains the origin, and one in terms of the Airy function for $x$ in an interval containing the transition near the largest zero of $L_{n}^{\alpha}(x)$. In this section we give an asymptotic expansion of $L_{n}^{\alpha}(n x)$ in terms of $L_{n}^{1 / 2}(n x)$, which in fact is an Hermite polynomial. We consider $x>1$ and for these values the expansion is convergent and is in particular of interest because this is the interval that contains the large zeros and the transition point at $x=4$.

When the parameter $\alpha$ of the Laguerre polynomial is large the asymptotic behavior can be described in terms of Hermite polynomials (see [12]). For example, we have the limit

$$
\lim _{\alpha \rightarrow \infty} \alpha^{-n / 2} L_{n}^{\alpha}(x \sqrt{\alpha}+\alpha)=\frac{(-1)^{n} 2^{-n / 2}}{n !} H_{n}(x / \sqrt{2}) .
$$

In [8] we have extended this limit by giving an asymptotic representation for large $\alpha$ and $n$ fixed in terms of Hermite polynomials. For more details on large $\alpha$ asymptotics we refer to [12]. The approach in this section is quite different because we take $\alpha$ fixed and $n$ large.

From (4.3) we obtain

$$
L_{n}^{(\alpha)}(n x)=\frac{1}{2 \pi i} \int_{\Gamma} f(w) \frac{e^{n \varphi(x, w)}}{(1-w)^{3 / 2}} \frac{d w}{w},
$$

where

$$
\varphi(x, w) \equiv \frac{x w}{w-1}-\log w, \quad f(w) \equiv(1-w)^{1 / 2-\alpha} .
$$

The function $\varphi(x, w)$ has two conjugate saddle points:

$$
w^{ \pm}=1-\frac{x}{2} \pm \frac{i}{2} \xi, \quad \xi=\sqrt{x(4-x)} .
$$

The square root defining $\xi$ is positive for $0<x<4$; for $x \geq 4$ we define $\xi=i \sqrt{x(x-4)}$, again with positive square root. In the expansion of the Laguerre polynomials we allow that the saddle points coalesce. 


\subsection{Construction of the expansion}

The function $f(w)$ of $(4.5)$ is analytic in $\Omega=\mathbb{C} \backslash[1, \infty)$ and we can expand $f(w)$ in in a two-point Taylor expansion at the two saddle points $w^{ \pm}$, using a slightly different form of $(2.3)$,

$$
f(w)=\sum_{k=0}^{\infty}\left[A_{k}+B_{k} w\right]\left(w-w^{+}\right)^{k}\left(w-w^{-}\right)^{k}
$$

After substituting expansion (4.8) in (4.5) and interchanging summation and integration we obtain

$$
L_{n}^{(\alpha)}(x n)=\sum_{k=0}^{\infty}\left[A_{k} \Phi_{k}(x, n)+B_{k} \Psi_{k}(x, n)\right]
$$

where

$$
\Phi_{k}(x, n)=\frac{1}{2 \pi i} \int_{\Gamma}\left(w-w^{+}\right)^{k}\left(w-w^{-}\right)^{k} \frac{e^{n \varphi(x, w)}}{(1-w)^{3 / 2}} \frac{d w}{w}
$$

and

$$
\Psi_{k}(x, n)=\frac{1}{2 \pi i} \int_{\Gamma}\left(w-w^{+}\right)^{k}\left(w-w^{-}\right)^{k} \frac{e^{n \varphi(x, w)}}{(1-w)^{3 / 2}} d w
$$

We have

$$
\begin{aligned}
& \Phi_{0}(x, n) \equiv L_{n}^{(1 / 2)}(n x)=\frac{(-1)^{n}}{n ! 2^{2 n+1} \sqrt{n x}} H_{2 n+1}(\sqrt{n x}), \\
& \Psi_{0}(x, n) \equiv L_{n-1}^{(1 / 2)}(n x)=\frac{(-1)^{n-1}}{(n-1) ! 2^{2 n-1} \sqrt{n x}} H_{2 n-1}(\sqrt{n x}),
\end{aligned}
$$

and, for $k=1,2,3, \ldots$,

$$
\Phi_{k}(x, n) \equiv \sum_{j=0}^{k}\left(\begin{array}{c}
k \\
j
\end{array}\right) x^{k-j} L_{n-k+j}^{(1 / 2-2 j)}(n x), \quad \Psi_{k}(x, n) \equiv \sum_{j=0}^{k}\left(\begin{array}{c}
k \\
j
\end{array}\right) x^{k-j} L_{n-k+j-1}^{(1 / 2-2 j)}(n x) .
$$

The sequences $\left\{\Phi_{k}(x, n)\right\}$ and $\left\{\Psi_{k}(x, n)\right\}, k=0,1,2, \ldots$, satisfy the recurrences

$$
\begin{gathered}
\Phi_{k}=\frac{1}{n-2 k+3 / 2}\left\{a_{1} \Phi_{k-1}+a_{2} \Phi_{k-2}+b_{1} \Psi_{k-1}+b_{2} \Psi_{k-2}\right\}, \\
a_{1}=(k-1)\left(x^{2}-2 x-2\right)-\frac{1}{2}, \quad a_{2}=(k-1) x(2-x), \\
b_{1}=(k-1)(2-3 x)+\frac{1-x}{2}, \quad b_{2}=(k-1) x\left(4 x-x^{2}-2\right), \\
\Psi_{k}=\frac{1}{n-2 k+1 / 2}\left\{c_{0} \Phi_{k}+c_{1} \Phi_{k-1}+c_{2} \Phi_{k-2}+d_{1} \Psi_{k-1}+d_{2} \Psi_{k-2},\right\},
\end{gathered}
$$




$$
\begin{aligned}
& c_{0}=(2-3 k) x+2(k-1)+\frac{1-x}{2}, \\
& c_{1}=(1-k) x^{3}+4(k-1) x^{2}+k x+2(1-k)+\frac{x-1}{2}, \quad c_{2}=-b_{2}, \\
& d_{1}=(4 k-3) x^{2}+2(4-5 k) x+2(k-1)+\frac{x^{2}-3 x+1}{2}, \\
& d_{2}=(k-1) x\left(x^{3}-6 x^{2}+9 x-2\right) .
\end{aligned}
$$

To verify the recursions (4.14) and (4.15) we write

$$
\begin{gathered}
\Phi_{k}(x, n)=-\frac{1}{2 \pi i n} \int_{C}\left(w-w^{+}\right)^{k-1}\left(w-w^{-}\right)^{k-1} \sqrt{1-w} \frac{\partial e^{n \varphi(x, w)}}{\partial w} d w, \\
\Psi_{k}(x, n)=-\frac{1}{2 \pi i n} \int_{C}\left(w-w^{+}\right)^{k-1}\left(w-w^{-}\right)^{k-1} w \sqrt{1-w} \frac{\partial e^{n \varphi(x, w)}}{\partial w} d w \\
\Psi_{k-1}(x, n)-w^{+} \Phi_{k-1}(x, n)=\frac{1}{2 \pi i} \int_{C}\left(w-w^{+}\right)^{k}\left(w-w^{-}\right)^{k-1} \frac{e^{n \varphi(x, w)}}{(1-w)^{3 / 2}} \frac{d w}{w}
\end{gathered}
$$

and

$$
\Psi_{k-1}(x, n)-w^{-} \Phi_{k-1}(x, n)=\frac{1}{2 \pi i} \int_{C}\left(w-w^{+}\right)^{k-1}\left(w-w^{-}\right)^{k} \frac{e^{n \varphi(x, w)}}{(1-w)^{3 / 2}} \frac{d w}{w} .
$$

Integrating by parts in (4.16) and (4.17), using (4.18) and (4.19) and after straightforward manipulations we obtain (4.14) and (4.15). Formulas (4.12)and (4.13) follow from (4.10) and (4.11) after simple calculations.

TheOREM 4.1. Expansion (4.9) is convergent, uniformly for $\alpha \in \mathbb{C}$ in compact sets, and $x \geq 1+\delta>1$. Moreover, $\left\{\Phi_{k}(x, n)\right\}$ and $\left\{\Psi_{k}(x, n)\right\}$ are asymptotic sequences for large $n$ :

$$
\begin{aligned}
& \Phi_{k}(x, n)=\mathcal{O}\left(n^{-\lfloor(k+1) / 2\rfloor}\right)\left[\left|\Phi_{0}(x, n)\right|+\left|\Psi_{0}(x, n)\right|\right], \\
& \Psi_{k}(x, n)=\mathcal{O}\left(n^{-\lfloor(k+1) / 2\rfloor}\right)\left[\left|\Phi_{0}(x, n)\right|+\left|\Psi_{0}(x, n)\right|\right],
\end{aligned}
$$

as $n \rightarrow \infty$ and $k=0,1,2, \ldots$.

Proof. We apply Proposition 2.2. Expansion (4.8) is uniformly convergent for $w$ inside the Cassini oval with foci $w^{+}$and $w^{-}$and "radius" $r=\left|w_{1}-w^{+}\right|\left|w_{1}-w^{-}\right|$, where $w_{1}=1$ is the singular point of $f$. Using (4.7) it follows that $r=x$. The points $w$ are inside the Cassini oval if they satisfy $\left|w-w^{+}\right|\left|w-w^{-}\right|<r=x$. Because $w^{+} w^{-}=1$, the origin $w=0$ is inside the oval only if $x>1$. Hence, the contour $\Gamma$ of (4.5) can be taken completely inside the oval only if $x>1$ (see also Figure 4.2). This proves the convergence of (4.9) for $x>1$. The asymptotic behavior in (4.20) follows from (4.12) and the recursions (4.14) and (4.15). More detailed asymptotic information can be obtained from the integrals in (4.10) and (4.11). 

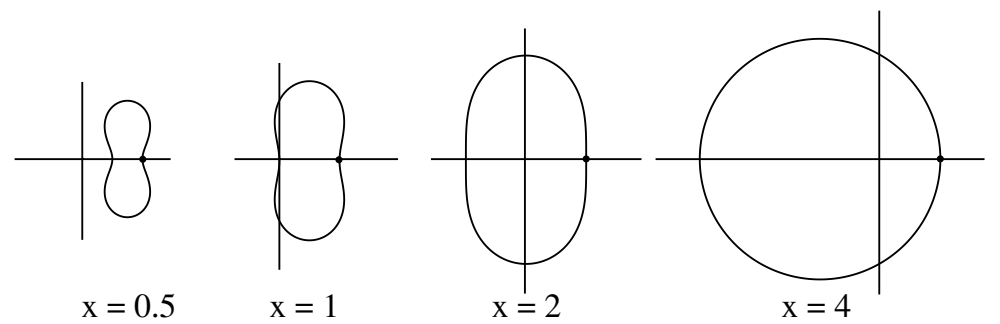

$\mathrm{x}=0.5$

$\mathrm{x}=1$

$\mathrm{x}=2$

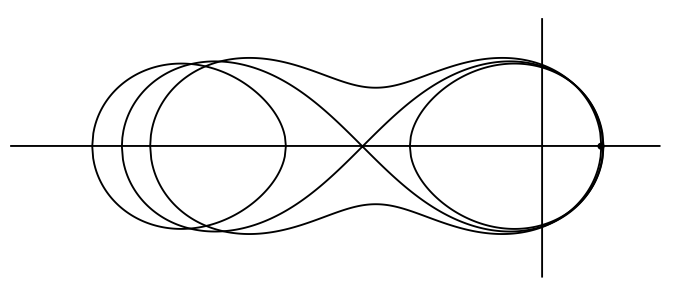

$\mathrm{x}=7.5, \quad \mathrm{x}=8$ (lemniscate) and $\mathrm{x}=8.5$

Figure 4.2. Cassini ovals for the expansion (4.8) for several values of $x$. For $w$ inside the ovals, the expansion is convergent. For $0<x<1$ the origin is outside the oval; for $x=4$ it is a circle, for $x=8$ a lemniscate. For $x>8$ the oval splits up into two parts. All ovals go through the point $w=1$, a singular point of $f(w)$.

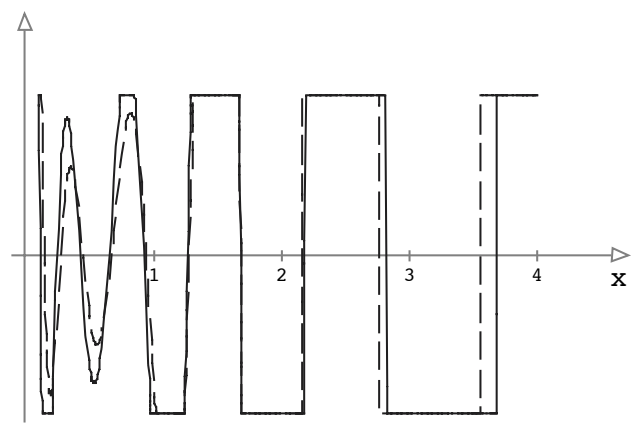

(a)

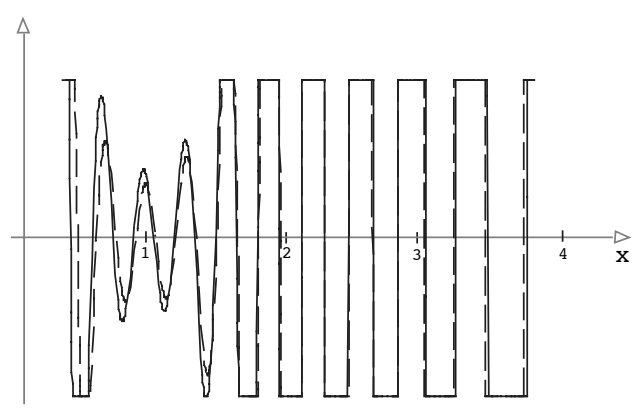

(b)

FiguRE 4.3. Numerical experiments on the approximation of Theorem 4.2 for large $n$ and $x \in(0,4]$. Continuous lines represent the Laguerre polynomial $L_{n}^{(4)}(n x)$ for (a) $n=10$ and (b) $n=20$. Dashed lines represent the first order approximation given by $A_{0} \Phi_{0}(x, n)+$ $B_{0} \Psi_{0}(x, n)$. Both graphics are cut for extreme values of the polynomials.

REMARK 4.4. The expansion in (4.9) has a meaning for all complex $x$, and has for all fixed $x$ an asymptotic meaning. The expansion is uniformly convergent for $|x| \geq 1+\delta>$ 1.

\subsection{Details on the coefficients}

The expressions (2.5) and (2.6) for the coefficients in (2.3) can be used in the present case also. We first write

$$
f(w)=\sum_{k=0}^{\infty}\left[a_{k}\left(w-w^{-}\right)+a_{k}^{\prime}\left(w-w^{+}\right)\right]\left[\left(w-w^{-}\right)\left(w-w^{+}\right)\right]^{k},
$$


and compare this with (4.8). By comparing coefficients of equal powers, it follows that $A_{k}$ and $B_{k}$ can be expressed in terms of $a_{k}$ and $a_{k}^{\prime}$. We have for $k=0,1,2, \ldots$

$$
A_{k}=-a_{k} w^{+}-a_{k}^{\prime} w^{-}, \quad B_{k}=a_{k}+a_{k}^{\prime} .
$$

We have

$$
A_{k} \equiv-2 \Re\left(w^{+} a_{k}\right), \quad B_{k} \equiv 2 \Re\left(a_{k}\right), \quad a_{0}=i\left(1-w^{-}\right)^{1 / 2-\alpha} \xi^{-1},
$$

and, for $k=1,2,3, \ldots$,

$$
a_{k}=\sum_{j=0}^{k} \frac{(k+j-1) !(\alpha-1 / 2)_{k-j}}{k ! j !(k-j) !(i \xi)^{k+j+1}}\left\{\frac{(-1)^{j} j}{\left(1-w^{+}\right)^{\alpha+k-j-1 / 2}}-\frac{(-1)^{k} k}{\left(1-w^{-}\right)^{\alpha+k-j-1 / 2}}\right\} .
$$

The coefficients can also be computed from the recursion relations

$$
\begin{aligned}
& x(k+1) A_{k+1}-x(k+1) B_{k+1}=(\alpha-1 / 2+2 k) A_{k}-(x k+1) B_{k}, \\
& x(k+1) A_{k+1}-x(x-3)(k+1) B_{k+1}=(\alpha+1 / 2+2 k) B_{k},
\end{aligned}
$$

where $k=0,1,2, \ldots$ Let, for $1<x \leq 4, x=4 \sin ^{2}(\theta / 2)$. Then $w^{ \pm}=e^{ \pm i \theta}$, and

$$
A_{0}=-2^{\beta+1} \sin ^{\beta}(\theta / 2) \cos [(\theta-\pi) \beta / 2-\theta], \quad B_{0}=2^{\beta+1} \sin ^{\beta}(\theta / 2) \cos [(\theta-\pi) \beta / 2] .
$$

where $\beta=1 / 2-\alpha$. This gives real expressions for the first coefficients to start the recursion relations in (4.24). For $x \geq 4$ we can obtain expressions in terms of hyperbolic functions by writing $x=4 \cosh ^{2}(\theta / 2)$, which gives $w^{ \pm}=-e^{ \pm \theta}$ and

$$
A_{0}=-2^{\beta+1} \cosh ^{\beta}(\theta / 2) \cosh \left[(\theta(\beta / 2-1)], \quad B_{0}=2^{\beta+1} \cosh ^{\beta}(\theta / 2) \cosh (\theta \beta / 2) .\right.
$$

\subsection{An alternative form of the expansion}

By using in (4.5) the substitution

$$
f(w)=\alpha_{0}+\beta_{0} w+\left(w-w^{-}\right)\left(w-w^{+}\right) g_{0}(w),
$$

where $\alpha_{0}$ and $\beta_{0}$ follow from substituting $w=w^{ \pm}$, we obtain by integrating by parts

$$
L_{n}^{(\alpha)}(n x)=\alpha_{0} \Phi_{0}(x, n)+\beta_{0} \Psi_{0}(x, n)+\frac{1}{2 \pi i n} \int_{\Gamma} f_{1}(w) \frac{e^{n \varphi(x, w)}}{(1-w)^{3 / 2}} \frac{d w}{w},
$$

where

$$
f_{1}(w)=w(1-w)^{3 / 2} \frac{d}{d w}\left[\left(\sqrt{1-w} g_{0}(w)\right]\right.
$$


Continuing this procedure we obtain the expansion in negative powers of $n$ :

$$
L_{n}^{(\alpha)}(n x)=\Phi_{0}(x, n) \sum_{k=0}^{\infty} \frac{\alpha_{k}}{n^{k}}+\Psi_{0}(x, n) \sum_{k=0}^{\infty} \frac{\beta_{k}}{n^{k}},
$$

where $\alpha_{k}$ and $\beta_{k}$ follow from

$$
f_{k}\left(w^{-}\right)=\alpha_{k}+\beta_{k} w^{-}, \quad f_{k}\left(w^{+}\right)=\alpha_{k}+\beta_{k} w^{+},
$$

where with $f_{0}(w)=f(w)$ and for $k=0,1,2, \ldots$

$$
\begin{aligned}
f_{k}(w) & =\alpha_{k}+\beta_{k} w+\left(w-w^{-}\right)\left(w-w^{+}\right) g_{k}(w), \\
f_{k+1}(w) & =w(1-w)^{3 / 2} \frac{d}{d w}\left[\left(\sqrt{1-w} g_{k}(w)\right] .\right.
\end{aligned}
$$

The expansion in (4.28) also follows from re-arranging expansion (4.9) by using the recursion relations for $\Phi_{k}(x, n)$ and $\Psi_{k}(x, n)$ in (4.14) and (4.15).

\begin{tabular}{|l|r|r|r|r|r|r|r|}
\hline$n$ & $L_{n}^{(1)}(3.5 n)$ & $\mathbf{L}_{n}^{(1)}(3.5,0)$ & $\mathbf{L}_{n}^{(1)}(3.5,1)$ & $\mathbf{L}_{n}^{(1)}(3.5,2)$ & $\mathbf{L}_{n}^{(1)}(3.5,3)$ & $\mathbf{L}_{n}^{(1)}(3.5,4)$ & $\mathbf{L}_{n}^{(1)}(3.5,5)$ \\
\hline 10 & 0.340506 & 0.343249 & 0.341724 & 0.340495 & 0.340449 & 0.340490 & 0.340504 \\
30 & -8.94039 & -8.86531 & -9.03530 & -8.95798 & -8.94213 & -8.94045 & -8.94038 \\
50 & -5.05678 & -5.05941 & -5.06764 & -5.05801 & -5.05689 & -5.05680 & -5.05678 \\
90 & 6.56556 & 6.56328 & 6.57572 & 6.56601 & 6.56547 & 6.56553 & 6.56556 \\
\hline
\end{tabular}

TABLE 4.5. Numerical experiment on the convergence rate of expansion (4.9) for $x=$ 3.5 and $a=1$. Here, $\mathbf{L}_{n}^{(\alpha)}(x, N) \equiv \sum_{k=0}^{N}\left[A_{k} \Phi_{k}(x, n)+B_{k} \Psi_{k}(x, n)\right]$, represents the truncated series in (4.9). All the rows are multiplied by an appropriate constant in order to keep the numbers small.

\section{Asymptotic expansions of Jacobi polynomials in terms of Chebyshev poly- nomials}

The large $n$ asymptotics for the Jacobi polynomials is discussed in [[3], Vol. II, §10.14], in particular for $x \in(-1,1)$. For $x$ bounded away from the points \pm 1 elementary functions (sine and cosine functions) can be used for describing the asymptotics. For $x$ close to \pm 1 Bessel functions can be used (Hilb-type formulas).

In this section we develop a convergent expansion that is valid for $x \in(-1,1)$ and the terms of the expansion constitute asymptotic scales for large $n$. It is possible to extend the results to complex values of $x$, but this will not be considered here. The first approximants are Chebyshev polynomials, which in fact are elementary functions, and the other terms can be obtained from recursions that show the asymptotic property. 


\subsection{Construction of the expansion}

Starting point is the integral representation that follows from [[3], Vol. II, p. 172]:

$$
P_{n}^{(\alpha, \beta)}(x)=\frac{1}{2 \pi i} \frac{(-1)^{n}}{2^{n}} \int_{\Gamma} \frac{(1-w-x)^{\alpha}(1+w+x)^{\beta}}{(1-x)^{\alpha}(1+x)^{\beta}} e^{n \varphi(x, w)} \frac{d w}{w},
$$

where we consider $x \in(-1,1)$; the function $\varphi(x, w)$ is defined by

$$
\varphi(x, w) \equiv \log (1+w+x)+\log (1-w-x)-\log w
$$

and $\Gamma$ is a simple closed contour, in the positive sense, around $w=0$. The points $w=-x \pm 1$ are outside the contour, and $(1-w-x)^{\alpha} /(1-x)^{\alpha}$ and $(1+w+x)^{\beta} /(1+x)^{\beta}$ are to be taken as unity when $w=0$.

The function $\varphi(x, w)$ has two conjugate saddle points:

$$
w^{ \pm}= \pm w_{0}, \quad w_{0}=i \sqrt{1-x^{2}} .
$$

We expand the integral by using the function

$$
f(w) \equiv(1-x)^{-\alpha-\frac{1}{2}}(1+x)^{-\beta-\frac{1}{2}}(1-w-x)^{\alpha+\frac{1}{2}}(1+w+x)^{\beta+\frac{1}{2}},
$$

which is analytic in

$$
\mathbb{C} \backslash\{(-\infty,-1-x] \bigcup[1-x, \infty)\} .
$$

We expand, using a slightly different form of (2.3),

$$
f(w)=\sum_{k=0}^{\infty}\left[A_{k}+B_{k} w\right]\left(w^{2}-w_{0}^{2}\right)^{k}
$$

where the coefficients $A_{k}$ and $B_{k}$ can be expressed in terms of the derivatives of $f(w)$ at $w= \pm w_{0}$; see the next subsection for more details.

After substituting expansion (5.3) in (5.1) and interchanging summation and integration we obtain

$$
P_{n}^{(\alpha, \beta)}(x)=\sum_{k=0}^{\infty}\left[A_{k} \Phi_{k}(x, n)+B_{k} \Psi_{k}(x, n)\right]
$$

where

$$
\begin{gathered}
\Phi_{k}(x, n)=\frac{(-1)^{n}}{2 \pi i} \frac{\sqrt{1-x^{2}}}{2^{n}} \int_{\Gamma} \frac{\left(w^{2}+1-x^{2}\right)^{k}}{W(x, w)} e^{n \varphi(x, w)} \frac{d w}{w} \\
\Psi_{k}(x, n)=\frac{(-1)^{n}}{2 \pi i} \frac{1}{2^{n} \sqrt{1-x^{2}}} \int_{\Gamma}\left(w^{2}+1-x^{2}\right)^{k} W(x, w) e^{n \varphi(x, w)} \frac{d w}{w},
\end{gathered}
$$


and

$$
W(x, w) \equiv \sqrt{(1-w-x)(1+w+x)} .
$$

We have

$$
\Phi_{0}(x, n) \equiv P_{n}^{(-1 / 2,-1 / 2)}(x), \quad \Psi_{0}(x, n) \equiv-\frac{1}{2}\left(1-x^{2}\right) P_{n-1}^{(1 / 2,1 / 2)}(x) .
$$

These Jacobi polynomials are Chebyshev polynomials:

$$
P_{n}^{(-1 / 2,-1 / 2)}(x)=\frac{2^{-2 n}(2 n) !}{(n !)^{2}} T_{n}(x), \quad P_{n}^{(1 / 2,1 / 2)}(x)=\frac{2^{-2 n}(2 n+1) !}{n !(n+1) !} U_{n}(x) .
$$

In terms of elementary functions:

$$
T_{n}(\cos \theta)=\cos n \theta, \quad U_{n}(\cos \theta)=\frac{\sin (n+1) \theta}{\sin \theta} .
$$

Furthermore

$$
\begin{gathered}
\Phi_{1}(x, n) \equiv \frac{1-x^{2}}{4(n+1)}\left[4(n+1) \Phi_{0}(x, n)-4 x(n-1) \Psi_{0}(x, n)+(2 n-1) \Psi_{0}(x, n-1)\right], \\
\Psi_{1}(x, n) \equiv \frac{-3\left(1-x^{2}\right)}{4(n+1)(n+2)}\left\{2\left[n+1-2 x^{2} n\right] \Psi_{0}(x, n)+x(2 n-1) \Psi_{0}(x, n-1)\right\}
\end{gathered}
$$

and, for $k=2,3,4, \ldots$,

$$
\begin{gathered}
\Phi_{k}(x, n) \equiv \sum_{j=0}^{k}\left(\begin{array}{c}
k \\
j
\end{array}\right) \frac{\left(1-x^{2}\right)^{k+j}}{4^{j}} P_{n-2 j}^{(2 j-1 / 2,2 j-1 / 2)}(x), \\
\Psi_{k}(x, n) \equiv-\frac{1}{2}\left(1-x^{2}\right) \sum_{j=0}^{k}\left(\begin{array}{c}
k \\
j
\end{array}\right) \frac{\left(1-x^{2}\right)^{k+j}}{4^{j}} P_{n-1-2 j}^{(2 j+1 / 2,2 j+1 / 2)}(x) .
\end{gathered}
$$

The sequences $\left\{\Phi_{k}(x, n)\right\}$ and $\left\{\Psi_{k}(x, n)\right\}$ satisfy the recursion relations

$$
\begin{gathered}
\Phi_{k}=\frac{1}{n+2 k-1}\left[a_{1} \Phi_{k-1}+a_{2} \Phi_{k-2}+b_{1} \Psi_{k-1}+b_{2} \Psi_{k-2}\right], \\
\Psi_{k}=\frac{1}{n+2 k}\left[c_{0} \Phi_{k}+c_{1} \Phi_{k-1}+c_{2} \Phi_{k-2}+d_{1} \Psi_{k-1}+d_{2} \Psi_{k-2}\right],
\end{gathered}
$$

where

$$
\begin{aligned}
& a_{1}=\left(1-x^{2}\right)(6 k-5), \quad a_{2}=-4(k-1)\left(1-x^{2}\right)^{2}, \\
& b_{1}=-x(4 k-3), \quad b_{2}=4 x(k-1)\left(1-x^{2}\right), \\
& c_{0}=-x(4 k-1), \quad c_{1}=x\left(1-x^{2}\right)(8 k-5), \quad c_{2}=-4 x\left(1-x^{2}\right)^{2}(k-1), \\
& d_{1}=3(2 k-1)\left(1-x^{2}\right), \quad d_{2}=-4\left(1-x^{2}\right)^{2}(k-1) .
\end{aligned}
$$


For the relations between Jacobi and Chebyshev polynomials we refer to [[13] p. 152153]. The expressions in (5.11) and (5.12) follow from contiguous relations of the Jacobi polynomials (see[[13] p. 166]).

To verify the recursions in (5.15) and (5.16), we write

$$
\begin{gathered}
\Phi_{k}(x, n)=\frac{-1}{2 \pi i} \frac{(-1)^{n}}{n 2^{n} \sqrt{1-x^{2}}} \int_{\Gamma}\left(w^{2}+1-x^{2}\right)^{k-1} W(x, w) \frac{\partial e^{n \varphi(x, w)}}{\partial w} d w \\
\Psi_{k}(x, n)=\frac{-1}{2 \pi i} \frac{(-1)^{n}}{n 2^{n} \sqrt{1-x^{2}}} \int_{\Gamma} w\left(w^{2}+1-x^{2}\right)^{k-1} W(x, w) \frac{\partial e^{n \varphi(x, w)}}{\partial w} d w
\end{gathered}
$$

Integrating by parts in (5.17) and (5.18) and after straightforward manipulations we obtain (5.15) and (5.16). The asymptotic behavior pointed out above follows from the definition of the Jacobi polynomials and the recurrences (5.15) and (5.16).

Theorem 5.1. Expansion (5.4) is convergent for $x \in(-1,1)$. Moreover, $\left\{\Phi_{k}(x, n)\right\}$ and $\left\{\Psi_{k}(x, n)\right\}$ are asymptotic sequences for large $n$ :

$$
\Phi_{k}(x, n)=\mathcal{O}\left(n^{-\lfloor(k+1) / 2\rfloor}\right), \quad \Psi_{k}(x, n)=\mathcal{O}\left(n^{-\lfloor(k+1) / 2\rfloor}\right),
$$

as $n \rightarrow \infty$ and $k=0,1,2, \ldots$.

Proof. Expansion (5.3) is uniformly convergent for $w$ inside the Cassini oval of focus $w^{ \pm}$and "radius"

$$
r=\min \left\{\left|1+x+w^{+}\right|\left|1+x+w^{-}\right|,\left|1-x+w^{+}\right|\left|1-x+w^{-}\right|\right\}=2(1-|x|) .
$$

Hence, (5.3) is convergent for $w \in \mathbb{C}$ such that $\left|w^{2}+1-x^{2}\right|<2(1-|x|)$. From Proposition 2.2 it follows that expansion (5.4) is convergent for $1-x^{2}=\left|w^{+} w^{-}\right|<2(1-|x|)$, that is, $\forall x \in(-1,1)$. The asymptotic property follows from the recursion relations in (5.15) and (5.16).

\subsection{Details on the coefficients}

The expressions for (2.5) and (2.6) for the coefficients in (2.3) can be used in the present case also. We first write

$$
f(w)=\sum_{k=0}^{\infty}\left[a_{k}\left(w-w_{0}\right)+a_{k}^{\prime}\left(w+w_{0}\right)\right]\left(w^{2}-w_{0}^{2}\right)^{k}
$$

and compare this with (5.3). By comparing coefficients of equal powers, it follows that $A_{k}$ and $B_{k}$ can be expressed in terms of $a_{k}$ and $a_{k}^{\prime}$. We have for $k=0,1,2, \ldots$

$$
A_{k}=\left(a_{k}^{\prime}-a_{k}\right) w_{0}, \quad B_{k}=a_{k}+a_{k}^{\prime} .
$$


After straightforward manipulations we find

$$
A_{k} \equiv \frac{2 \Im\left[a_{k} \sqrt{1-x^{2}}\right]}{(1-x)^{\alpha+1 / 2}(1+x)^{\beta+1 / 2}} \quad \text { and } \quad B_{k} \equiv \frac{2 \Re a_{k}}{(1-x)^{\alpha+1 / 2}(1+x)^{\beta+1 / 2}}
$$

where

$$
a_{0}=-\frac{\left(1-x+w_{0}\right)^{\alpha+1 / 2}\left(1+x-w_{0}\right)^{\beta+1 / 2}}{2 w_{0}}
$$

and, for $m=1,2,3, \ldots$,

$$
\begin{aligned}
a_{m}= & \frac{1}{m !} \sum_{k=0}^{m} \frac{(m+k-1) !}{k !(m-k) !\left(2 w_{0}\right)^{m+k+1}} \times \\
& \sum_{j=0}^{m-k}\left(\begin{array}{c}
m-k \\
j
\end{array}\right)\left\{\frac{k(-1)^{m+j}(-\alpha-1 / 2)_{j}(-\beta-1 / 2)_{m-k-j}}{\left(1-x-w_{0}\right)^{j-\alpha-1 / 2}\left(1+x+w_{0}\right)^{m-k-j-\beta-1 / 2}}-\right. \\
& \left.\frac{m(-1)^{k+j}(-\alpha-1 / 2)_{j}(-\beta-1 / 2)_{m-k-j}}{\left(1-x+w_{0}\right)^{j-\alpha-1 / 2}\left(1+x-w_{0}\right)^{m-k-j-\beta-1 / 2}}\right\} .
\end{aligned}
$$

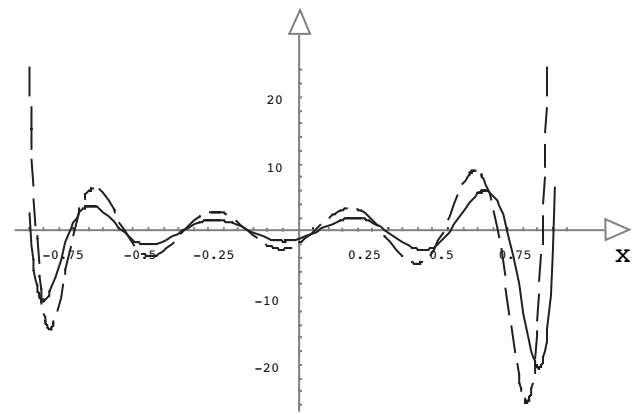

(a)

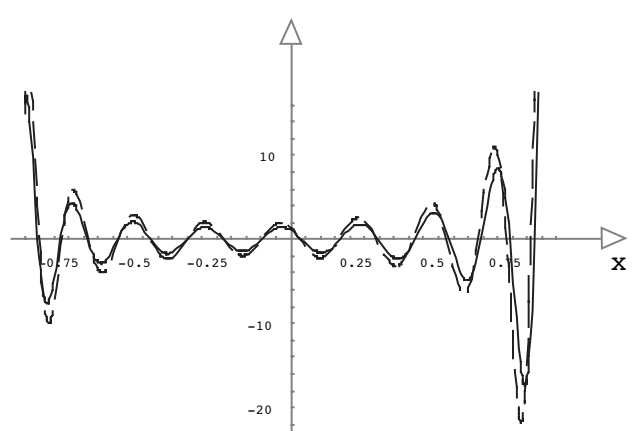

(b)

FigURE 5.2. Numerical experiments about the approximation of Theorem 5.1 for large $n$ and $-1<x<1$. Continuous lines represent the Jacobi polynomial $P_{n}^{(3,4)}(x)$ for (a) $n=10$ and (b) $n=20$. Dashed lines represent the first order approximation given by $A_{0} \Phi_{0}+B_{0} \Psi_{0}$.

\begin{tabular}{|l|r|l|l|l|l|l|l|}
\hline$n$ & $P_{n}^{(3 / 2,1 / 2)}(0)$ & $\mathbf{P}_{n}(0,0)$ & $\mathbf{P}_{n}(0,1)$ & $\mathbf{P}_{n}(0,2)$ & $\mathbf{P}_{n}(0,3)$ & $\mathbf{P}_{n}(0,4)$ & $\mathbf{P}_{n}(0,5)$ \\
\hline 10 & -0.336376 & -0.348029 & -0.348029 & -0.337153 & -0.336376 & -0.336340 & -0.336360 \\
30 & -0.201847 & -0.204304 & -0.204304 & -0.201909 & -0.201839 & -0.201845 & -0.201847 \\
50 & -0.157618 & -0.158781 & -0.158781 & -0.157636 & -0.157615 & -0.157617 & -0.157618 \\
90 & -0.118124 & -0.118612 & -0.118612 & -0.118128 & -0.118123 & -0.118124 & -0.118124 \\
\hline
\end{tabular}

TABLE 5.3. Numerical experiment on the convergence rate of expansion (5.4) for $x=0$, $\alpha=3 / 2$ and $\beta=1 / 2$. Here, $\mathbf{P}_{n}(x, N) \equiv \sum_{k=0}^{N}\left[A_{k} \Phi_{k}(x, n)+B_{k} \Psi_{k}(x, n)\right]$, represents the truncated series in (5.4). 


\section{A few other examples of convergent asymptotic expansions}

Consider the integral for the modified Bessel function (for properties of the special functions in this section we refer to [3] or [13])

$$
I_{\nu}(z)=\frac{(2 z)^{\nu} e^{z}}{\sqrt{\pi} \Gamma\left(\nu+\frac{1}{2}\right)} \int_{0}^{1} e^{-2 z t}[t(1-t)]^{\nu-\frac{1}{2}} d t
$$

The usual method for obtaining the asymptotic expansion for large $z$ consists of substituting the expansion $(1-t)^{\nu-\frac{1}{2}}=\sum\left(\begin{array}{c}\nu-\frac{1}{2} \\ k\end{array}\right)(-t)^{k}$ and by interchanging the order of summation and integration. The resulting integrals are not evaluated over $[0,1]$ but over $[0, \infty)$. This latter step gives the divergent asymptotic expansions. If we integrate over $[0,1]$ we obtain an expansion in terms of incomplete gamma functions:

$$
I_{\nu}(z)=\frac{e^{z}}{\sqrt{2 z \pi} \Gamma\left(\nu+\frac{1}{2}\right)} \sum_{k=0}^{\infty}(-1)^{k}\left(\begin{array}{c}
\nu-\frac{1}{2} \\
k
\end{array}\right) \frac{\gamma(\nu+k+1 / 2,2 z)}{(2 z)^{k}} .
$$

For fixed values of $z$ the incomplete gamma functions have the asymptotic behaviour

$$
\gamma(\nu+k+1 / 2,2 z)=\frac{e^{-2 z}(2 z)^{\nu+k+\frac{1}{2}}}{\nu+k+\frac{1}{2}}\left[1+\mathcal{O}\left(k^{-1}\right)\right], \quad k \rightarrow \infty
$$

and we see that the terms behave like $\mathcal{O}\left(k^{-\nu-3 / 2}\right)$. So, convergence is guaranteed if $\Re \nu>-\frac{1}{2}$. A further examination of the terms of the expansion shows that for large $z$ it is better to use the asymptotic property of the expansion (the ratio of successive terms is of order $\mathcal{O}(1 / z))$. The incomplete gamma functions can be computed by using a backward recusion scheme.

Another example is the $K$-Bessel function given by

$$
K_{\nu}(z)=\frac{\sqrt{\pi}(2 z)^{\nu} e^{-z}}{\Gamma\left(\nu+\frac{1}{2}\right)} \int_{0}^{\infty} e^{-2 z t}[t(1+t)]^{\nu-\frac{1}{2}} d t .
$$

Again expanding $(t+1)^{\nu-1 / 2}=\sum\left(\begin{array}{c}\nu-\frac{1}{2} \\ k\end{array}\right)(t)^{k}$ gives the standard expansion. A convergent expansion can be obtained by using

$$
(t+1)^{\nu-1 / 2}=\sum c_{k}\left(\frac{t}{t+1}\right)^{k}, \quad c_{k}=(-1)^{k}\left(\begin{array}{c}
\frac{1}{2}-\nu \\
k
\end{array}\right)
$$

This gives a convergent expansion in terms of confluent hypergeometric functions

$$
K_{\nu}(z)=\sqrt{\frac{\pi}{2 z}} e^{-z} \sum_{k=0}^{\infty}(-1)^{k} \frac{\left(\nu+\frac{1}{2}\right)_{k}\left(\nu-\frac{1}{2}\right)_{k}}{k !} U\left(k, \frac{1}{2}-\nu, 2 z\right) .
$$


We have

$$
U\left(0, \frac{1}{2}-\nu, 2 z\right)=1, \quad U\left(1, \frac{1}{2}-\nu, 2 z\right)=(2 z)^{\nu+\frac{1}{2}} e^{2 z} \Gamma\left(-\frac{1}{2}-\nu, 2 z\right),
$$

again an incomplete gamma function. Other $U$-functions can be obtained by recursion. For large $k$ the $U$-function behaves like [11]

$$
k ! U\left(k, \frac{1}{2}-\nu, 2 z\right)=\mathcal{O}\left(k^{\alpha} e^{-2 \sqrt{2 k z}}\right),
$$

where $\alpha$ is some constant. It follows that the convergence is better than in the previous example.

As a final example we consider an expansion of the Kummer function. Tricomi [14] has derived several convergent expansions of the ${ }_{1} F_{1}$-function in terms of Bessel functions that are useful for evaluating the function when the parameters are large. For example, we have [14]

$$
{ }_{1} F_{1}(a, c ; z)=e^{\frac{1}{2} z} \Gamma(c)(\kappa z)^{(1-c) / 2} \sum_{n=0}^{\infty} A_{n}\left(\kappa, \frac{1}{2} c\right)\left(\frac{z}{4 \kappa}\right)^{n / 2} J_{c-1+n}(2 \sqrt{\kappa z}),
$$

where $\kappa=\frac{1}{2} c-a$ and the $A_{n}(\kappa, \lambda)$ are coefficients in the generating function

$$
e^{2 \kappa z}(1-z)^{\kappa-\lambda}(1+z)^{-\kappa-\lambda}=\sum_{n=0}^{\infty} A_{n}(\kappa, \lambda) z^{n}
$$

The series in (6.8) is convergent in the entire $z$-plane. Moreover, it can be used for the evaluation of ${ }_{1} F_{1}(a, c ; z)$ for large $\kappa$, because the series has an asymptotic property. For further details on these expansions we refer to [14].

\section{Acknowledgements}

The authors thank the referee for the comments on the first version of the paper.

J. L. López wants to thank the C.W.I. of Amsterdam for its scientific and financial support during the realization of this work. The financial support of the saving bank Caja Rural de Navarra is also acknowledged. 


\section{References}

[1] Rui Bo And R. Wong, Uniform asymptotic expansion of Charlier polynomials. Methods Appl. Anal. 1 (1994), 294-313.

[2] T. M. Dunster, Uniform asymptotic expansion for Charlier polynomials. J. Approx. Theor. 112 (1994), 93-133.

[3] A. Erdélyi, Higher transcendental functions, Vols. I, II, III, A. Erdélyi, ed. McGraw-Hill (Reprinted by Krieger Publishing Co., Malabar, FL, 1981.) (1953).

[4] C.L. Frenzen and R. Wong, Uniform asymptotic expansions of Laguerre polynomials, SIAM J. Math. Anal., 19 (1988), 1232-1248.

[5] W.M.Y. Goн, Plancherel-Rotach asymptotics for the Charlier polynomials. Constructive Approximation 14(1998), 151-168.

[6] L.C. Hsu, Certain asymptotic expansions for Laguerre polynomials and Charlier polynomials. Approx. Theory Appl. (N.S.) 11 (1995), 94-104.

[7] R. Koekoek and R. F. Swarttouw, Askey scheme of hypergeometric orthogonal polynomials and its q-analogue, http://aw.twi.tudelft.nl/ ºekoek/askey.html, (1999).

[8] J. L. Lopez and N.M. Temme, Approximations of orthogonal polynomials in terms of Hermite polynomials. Methods Appl. Anal. 6 (1999), 131-146.

[9] J. L. Lopez and N.M. Temme, Two-point Taylor expansions of analytic functions. Stud. Appl. Math, 109 (2002), 297-311.

[10] José L. Lopez and Nico M. Temme, Multi-point Taylor expansions of analytic functions. Submitted for publication.

[11] Y. L. Luke, The Special Functions and their Applications, Academic Press, New York (1969).

[12] N.M. Temme, Asymptotic estimates for Laguerre polynomials, ZAMP 41 (1990), 114-126.

[13] N.M. Temme, Special Functions. An Introduction to the Classical Functions of Mathematical Physics, John Wiley and Sons, New York (1996).

[14] F.G. Tricomi, Funzioni ipergeometriche confluenti, Edizione Cremonese, Roma (1954)

[15] R. Wong, Asymptotic Approximations of Integrals, Academic Press, New York (1989). Reprinted by SIAM in 2001. 